\title{
Counting subwords in a partition of a set
}

\author{
Toufik Mansour \\ Department of Mathematics, University of Haifa, 31905 Haifa, Israel \\ toufik@math.haifa.ac.il \\ Mark Shattuck \\ Department of Mathematics, University of Tennessee, Knoxville, TN 37996 \\ shattuck@math.utk. edu \\ Sherry H.F. Yan* \\ Department of Mathematics, Zhejiang Normal University, 321004 Jinhua, P.R. China \\ huifangyan@hotmail.com
}

Submitted: Sep 22, 2009; Accepted: Jan 15, 2010; Published: Jan 22, 2010

Mathematics Subject Classification: 05A18, 05A15, 05A05, 68R05

\begin{abstract}
A partition $\pi$ of the set $[n]=\{1,2, \ldots, n\}$ is a collection $\left\{B_{1}, \ldots, B_{k}\right\}$ of nonempty disjoint subsets of $[n]$ (called blocks) whose union equals $[n]$. In this paper, we find explicit formulas for the generating functions for the number of partitions of $[n]$ containing exactly $k$ blocks where $k$ is fixed according to the number of occurrences of a subword pattern $\tau$ for several classes of patterns, including all words of length 3. In addition, we find simple explicit formulas for the total number of occurrences of the patterns in question within all the partitions of $[n]$ containing $k$ blocks, providing both algebraic and combinatorial proofs.
\end{abstract}

\section{Introduction}

A partition of $[n]=\{1,2, \ldots, n\}$ is a decomposition of $[n]$ into non-overlapping subsets $B_{1}, B_{2}, \ldots, B_{k}$, called blocks, which are listed in increasing order of their least elements $(1 \leqslant k \leqslant n)$. We will represent a partition $\pi=B_{1}, B_{2}, \ldots, B_{k}$ in the canonical sequential form $\pi=\pi_{1} \pi_{2} \cdots \pi_{n}$ such that $j \in B_{\pi_{j}}, 1 \leqslant j \leqslant n$. Therefore, a sequence $\pi=\pi_{1} \pi_{2} \cdots \pi_{n}$ over the alphabet $[k]$ represents a partition of $[n]$ with $k$ blocks if and only if it is a restricted growth function of $[n]$ onto $[k]$ (see, e.g., $[11,13,14]$ for details). For instance,

${ }^{*}$ The third author was supported by the National Natural Science Foundation of China (no. 10901141). 
123214154 is the canonical sequential form of the partition $\{1,5,7\},\{2,4\},\{3\},\{6,9\}$, $\{8\}$ of [9]. Throughout this paper, partitions will be identified with their corresponding canonical sequences. The set of all partitions of $[n]$ with exactly $k$ blocks will be denoted by $P(n, k)$ and has cardinality given by the Stirling number of the second kind, denoted by $S_{n, k}$.

For positive integers $a$ and $b$, let $[a]^{b}$ denote the set of words of length $b$ in the alphabet $[a]$. Given any word $\alpha$ in $[k]^{n}$ possessing $m$ distinct letters, let $\operatorname{red}(\alpha)$ denote the member of $[m]^{n}$ gotten by replacing all letters corresponding to the smallest element occurring in $\alpha$ with 1, replacing all letters corresponding to the second smallest element of $\alpha$ with 2, and so on (often referred to as the reduction of $\alpha$ ). For example, if $n=8, m=4$, and $\alpha=25662856$, then $\operatorname{red}(\alpha)=12331423$.

Let $\tau$ denote a member of $[m]^{\ell}$ possessing at least one letter for each member of $[m]$. We will call such a word $\tau$ a subword pattern. We will say that there is an occurrence of $\tau=\tau_{1} \tau_{2} \cdots \tau_{\ell}$ at index $i$ in the word $\alpha=\alpha_{1} \alpha_{2} \cdots \alpha_{n}$ if $\operatorname{red}\left(\alpha_{i} \alpha_{i+1} \cdots \alpha_{i+\ell-1}\right)=\tau$. The number of occurrences of the subword $\tau$ in $\alpha$ is the number of indices $i, 1 \leqslant i \leqslant n-\ell+1$, for which $\operatorname{red}\left(\alpha_{i} \alpha_{i+1} \cdots \alpha_{i+\ell-1}\right)=\tau$. For example, if $\alpha=535251472$, then there are three occurrences of the $\tau=231$ in $\alpha$ (corresponding to 352, 251, and 472) and two occurrences of $\tau=212$ (corresponding to 535 and 525). Note that here we are requiring that the letters within some word corresponding to an occurrence of a subword pattern $\tau$ be consecutive.

Several authors have studied various properties of the set of partitions. For instance, Chen et al. [2], Klazar [7], Sagan [12], Mansour and Severini [9], and Jelínek and Mansour [6] have studied pattern avoiding partitions. In [8], Mansour and Munagi studied the number of partitions of $[n]$ according to the number of $\ell$-levels (the subword pattern $\left.11 \cdots 1 \in[1]^{\ell}\right), \ell$-rises (the subword pattern $12 \cdots \ell \in[\ell]^{\ell}$ ), and $\ell$-descents (the subword pattern $\left.\ell(\ell-1) \cdots 1 \in[\ell]^{\ell}\right)$. In this paper, we consider the problem of counting various subword patterns within the members of $P(n, k)$. This extends earlier work done in [1] on counting occurrences of various subword patterns within the members of $[k]^{n}$. Counting the number of permutations, words or compositions according to the number of occurrences of a subword pattern is a classical problem in combinatorics; see, e.g., Elizalde and Noy [3], Heubach and Mansour [4, 5], Mansour and Sirhan [10], and the references therein.

Given a subword pattern $\tau$ and a word $\alpha$, let $\tau(\alpha)$ denote the number of occurrences of $\tau$ within $\alpha$. We consider the general problem of finding an explicit formula for the ordinary generating function defined by

$$
F_{\tau}(x, y, k)=\sum_{n \geqslant 0} x^{n}\left(\sum_{\lambda \in P(n, k)} y^{\tau(\lambda)}\right),
$$

for various subword patterns $\tau$ where $k \geqslant 1$ is fixed. In the next section, we compute $F_{\tau}(x, y, k)$ for several general patterns of arbitrary length $\ell$, noting the particular case $\ell=3$. In the third section, we provide a complete solution to the problem when $\tau$ has length 3 and compute recurrences for the remaining cases, which are more difficult. In each case, we find a simple explicit formula for the total number of occurrences of $\tau$ within all the members of $P(n, k)$, providing both algebraic and combinatorial proofs. 


\section{Counting a subword pattern of length $\ell$}

In this section, we find $F_{\tau}(x, y, k)$ for several cases of $\tau$. This extends the work of Mansour and Munagi [8] who found $F_{\tau}(x, y, k)$ for the subword pattern $\tau=11 \cdots 1 \in[1]^{\ell}$ and proved the following theorem.

Theorem 2.1. The ordinary generating function for the number of partitions of $[n]$ with $k$ blocks according to the number occurrences of the subword pattern $\tau=11 \cdots 1 \in[1]^{\ell}$ is given by

$$
F_{\tau}(x, y, k)=\frac{\left(x+x^{2}+\cdots+x^{\ell-1}+x^{\ell} /(1-x y)\right)^{k}}{\prod_{j=0}^{k-1}\left(1-j\left(x+x^{2}+\cdots+x^{\ell-1}+x^{\ell} /(1-x y)\right)\right)}
$$

\subsection{The subword patterns $\tau=12 \cdots 22$ and $\tau=11 \cdots 12$}

In this section, we find the generating functions which correspond to the subword patterns $\tau=12 \cdots 22$ and $\tau=11 \cdots 12$. By the following theorem, we need only find one of these.

Theorem 2.2. For all positive integers $k$,

$$
F_{11 \cdots 12}(x, y, k)=F_{12 \cdots 22}(x, y, k) .
$$

Proof. To show this, first express the canonical representation $\alpha=\pi_{1} \pi_{2} \cdots \pi_{n}$ for each member of $P(n, k)$ as $\alpha=\alpha_{1} \alpha_{2} \cdots \alpha_{r}$, where each $\alpha_{i}, 1 \leqslant i \leqslant r$, is a non-empty, nondecreasing word (i.e., contains no descents) and where the largest (= last) member of the word $\alpha_{i}$ is larger than the smallest (= first) member of $\alpha_{i+1}$ for all $i$. Suppose that the set of distinct letters in $\alpha_{i}$ is $a_{1}<a_{2}<\cdots<a_{t}$ and that $\alpha_{i}=a_{i_{1}} a_{i_{2}} \cdots a_{i_{s}}$ for some positive integers $s$ and $t$ with $1 \leqslant i_{j} \leqslant t$ for each $j$. Let $\alpha_{i}^{\prime}$ be the word given by $\alpha_{i}^{\prime}=a_{t+1-i_{s}} a_{t+1-i_{s-1}} \cdots a_{t+1-i_{1}}$ and let $\alpha^{\prime}$ be the partition given by $\alpha^{\prime}=\alpha_{1}^{\prime} \alpha_{2}^{\prime} \cdots \alpha_{r}^{\prime}$. It may be verified that the mapping $\alpha \mapsto \alpha^{\prime}$ is a bijection of $P(n, k)$ which changes each occurrence of $11 \cdots 12$ to an occurrence of $12 \cdots 22$ (and each occurrence of $12 \cdots 22$ to one of $11 \cdots 12)$.

We now provide an explicit formula for $F_{\tau}(x, y, k)$ in the case when $\tau=12 \cdots 22$.

Theorem 2.3. Let $\tau=12 \cdots 22 \in[2]^{\ell}$ be a subword pattern. Then the generating function $F_{\tau}(x, y, k)$ is given by

$$
x^{k} \frac{\left(1-x^{\ell-2}(1-y)\right)^{k-1}}{\prod_{a=1}^{k}\left(1-x \sum_{j=0}^{a-1}\left(1-x^{\ell-1}(1-y)\right)^{j}\right)} .
$$

Proof. From Theorem 2.2 in [1], we have that the generating function $Q(x, y, k)$ for the number of words $\pi$ of length $n$ over the alphabet $[k]$ according to the number occurrences of $\tau=11 \cdots 12$ in $\pi$ is given by

$$
Q(x, y, k)=\frac{1}{1-x \sum_{i=0}^{k-1}\left(1-x^{\ell-1}(1-y)\right)^{i}} .
$$


This is also the generating function for the number of occurrences of $\tau=12 \cdots 22$ over such words (simply replace each letter $r$ with $k+1-r$ and reverse order). Let us consider the words $1 k \pi$, where $\pi$ is a word over the alphabet $[k]$, and consider whether or not the first letter of $\pi$ is $k$. This implies that the generating function for the number of words $k \pi$ of length $n$ over the alphabet $[k]$ according to the number occurrences of $12 \cdots 22$ in $1 k \pi$ is given by

$$
Q^{\prime}(x, y, k)=\left(x^{\ell-1} y+x\left(1-x^{\ell-2}\right)\right) Q(x, y, k)=\frac{x\left(1-x^{\ell-2}(1-y)\right)}{1-x \sum_{i=0}^{k-1}\left(1-x^{\ell-1}(1-y)\right)^{i}} .
$$

From the fact that each partition $\pi$ of $[n]$ with exactly $k$ blocks may be expressed uniquely as $\pi=1 \pi^{(1)} 2 \pi^{(2)} \cdots k \pi^{(k)}$ such that each $\pi^{(i)}$ is a word over the alphabet $[i]$, we have that the generating function $F_{\tau}(x, y, k)$ is given by $\frac{x}{1-x} \prod_{j=2}^{k} Q^{\prime}(x, y, j)$, which completes the proof.

A similar, though lengthier, argument may be given establishing $F_{\tau}(x, y, k)$ directly in the case $\tau=11 \cdots 12$. When $y=1$, the generating function in Theorem 2.3 reduces to that for the Stirling number of the second kind. Taking $\ell=3$ in Theorem 2.3 yields

$$
F_{122}(x, y, k)=x^{k} \frac{(1-x(1-y))^{k-1}}{\prod_{a=1}^{k}\left(1-x \sum_{j=0}^{a-1}\left(1-x^{2}(1-y)\right)^{j}\right)} .
$$

Differentiating the generating function in Theorem 2.3 yields

$$
\begin{aligned}
& \left.\frac{d}{d y} F_{\tau}(x, y, k)\right|_{y=1} \\
& =F_{\tau}(x, 1, k)\left(x^{\ell-2}(k-1)-\sum_{a=1}^{k} \frac{\left.\frac{d}{d y}\left(1-x \sum_{j=0}^{a-1}\left(1-x^{\ell-1}(1-y)\right)^{j}\right)\right|_{y=1}}{1-a x}\right) \\
& =F_{\tau}(x, 1, k)\left(x^{\ell-2}(k-1)+\sum_{a=1}^{k} \frac{x^{\ell}\left(\begin{array}{l}
a \\
2
\end{array}\right)}{1-a x}\right),
\end{aligned}
$$

which implies the following corollary to Theorem 2.3.

Corollary 2.4. The total number of occurrences of the subword $12 \cdots 22 \in[2]^{\ell}$ in all of the partitions of $[n]$ with exactly $k$ blocks is given by

$$
(k-1) S_{n-\ell+2, k}+\sum_{j=2}^{k}\left(\begin{array}{l}
j \\
2
\end{array}\right) f_{n, j}
$$

where $f_{n, j}=\sum_{i=\ell}^{n-k} j^{i-\ell} S_{n-i, k}$ and $S_{i, j}$ is the Stirling number of the second kind.

We now provide a combinatorial proof of Corollary 2.4. Occurrences of $\tau=12 \cdots 22$ in members of $P(n, k)$ in which the element corresponding to the first 2 starts a block are 
synonymous with occurrences of 12 in members of $P(n-\ell+2, k)$ in which the element corresponding to the 2 starts a block. To see this, note that if the element $i$ starts block $b, b \geqslant 2$, within a member $\lambda \in P(n-\ell+2, k)$, then one can increase all members of $[i+1, n-\ell+2]=\{i+1, i+2, \cdots, n-\ell+2\}$ by $\ell-2$ within $\lambda$, leaving them within their current blocks, and add the elements $i+1, i+2, \cdots, i+\ell-2$ to block $b$ to obtain an occurrence of $\tau=12 \cdots 22$ within a member of $P(n, k)$ in which the first 2 starts a block. There are clearly $(k-1) S_{n-\ell+2, k}$ occurrences of 12 in which the 2 starts a block within the members of $P(n-\ell+2, k)$ since they occur each time a new block is started (after the first). So to complete the proof, we must show that the total number of occurrences of $\tau$ in which the number corresponding to the 2 does not start a block is given by the sum in Corollary 2.4. We'll prove a more general result which we'll use in subsequent sections.

If $\lambda$ is a partition of $[n]$ and $i \in[n]$, then we will say that $i$ is minimal if $i$ is the smallest element of a block of $\lambda$, i.e., if the $i^{\text {th }}$ slot of the canonical representation of $\lambda$ corresponds to the first occurrence of a letter. Given $\lambda \in P(n, k)$ and $\tau$ a subword pattern, we'll call an occurrence of $\tau$ within $\lambda$ primary if no letter of $\tau$ corresponds to a minimal element of $\lambda$. The following lemma provides an explicit formula for the total number of primary occurrences of a subword $\tau$. Taking $\tau=12 \cdots 22$ completes the proof of Corollary 2.4.

Lemma 2.5. Let $\tau$ be any subword pattern of length $\ell$ in the alphabet $[m]$. Then the total number of primary occurrences of the subword $\tau$ in all of the partitions of $[n]$ with exactly $k$ blocks is given by

$$
\sum_{j=m}^{k}\left(\begin{array}{c}
j \\
m
\end{array}\right) f_{n, j}
$$

where $f_{n, j}=\sum_{i=\ell}^{n-k} j^{i-\ell} S_{n-i, k}$.

Proof. Given $i$ and $j$, where $\ell \leqslant i \leqslant n-k$ and $m \leqslant j \leqslant k$, consider all the members of $P(n, k)$ which may be decomposed uniquely as

$$
\pi=\pi^{\prime} j \alpha \beta
$$

where $\pi^{\prime}$ is a partition with $j-1$ blocks, $\alpha$ is a word of length $i$ in the alphabet $[j]$ whose last $\ell$ letters constitute an occurrence of $\tau$, and $\beta$ is possibly empty. For example, if $i=6, j=5, m=4, \tau=1243$, and $\pi=122324513135421 \in P(15,5)$, then $\pi^{\prime}=122324$, $\alpha=131354$, and $\beta=21$. Note that there are $\left(\begin{array}{c}j \\ m\end{array}\right)$ choices for the final $\ell$ letters of $\alpha$ since these letters are to form an occurrence of $\tau$ (the smallest member of $[j]$ chosen will correspond to the 1 in $\tau$, the second smallest to 2 , etc.). The total number of primary occurrences of $\tau$ can then be obtained by finding the number of partitions which may be expressed as in (1) for each $i$ and $j$ and then summing over all possible values of $i$ and $j$. And there are $\left(\begin{array}{c}j \\ m\end{array}\right) j^{i-\ell} S_{n-i, k}$ members of $P(n, k)$ which may be expressed as in (1) since one can pick the letters $\pi^{\prime} j \beta$ in $S_{n-i, k}$ ways (as they constitute a partition of an $n-i$ element set into $k$ blocks) and then insert $\alpha$ in $j^{i-\ell}\left(\begin{array}{c}j \\ m\end{array}\right)$ ways (as there are $j^{i-\ell}$ choices for the first $i-\ell$ letters of $\alpha$ and $\left(\begin{array}{c}j \\ m\end{array}\right)$ choices for the final $\ell$ letters). 


\subsection{The subword patterns $\tau=22 \cdots 21$ and $\tau=21 \cdots 11$}

In this section, we find $F_{\tau}(x, y, k)$ in the cases when $\tau=22 \cdots 21$ and $\tau=21 \cdots 11$. Again, we need only consider one case since the bijection used to establish Theorem 2.2 above also shows that the subword patterns $\tau=22 \cdots 21$ and $\tau=21 \cdots 11$ of the same length are identically distributed on $P(n, k)$, upon decomposing the canonical representations into non-increasing words (i.e., words which contain no rises) instead of decomposing them into non-decreasing words as before.

Theorem 2.6. For all positive integers $k$,

$$
F_{21 \cdots 11}(x, y, k)=F_{22 \cdots 21}(x, y, k) .
$$

We now provide an explicit formula for $F_{\tau}(x, y, k)$ in the case when $\tau=22 \cdots 21$.

Theorem 2.7. Let $\tau=22 \cdots 21 \in[2]^{\ell}$ be a subword pattern. Then the generating function $F_{\tau}(x, y, k)$ is given by

$$
x^{k}(y-1)^{k} \prod_{a=1}^{k} \frac{x^{\ell-2}\left(1-x^{\ell-1}(1-y)\right)^{a-1}}{1-x^{\ell-2}(1-y)-\left(1-x^{\ell-1}(1-y)\right)^{a}} .
$$

Proof. Let $G=G(x, y, a)$ and $G^{\prime}=G^{\prime}(x, y, a)$ denote the generating functions for the number of words $\pi$ of length $n$ over the alphabet $[a]$ according to the number of occurrences of $\tau=22 \cdots 21$ in $\pi$ and according to the number of occurrences of $\tau$ in $a \pi$, respectively, where $a \geqslant 2$. Let $G^{\prime \prime}=G^{\prime \prime}(x, y, a)$ denote the generating function for the number of words $\pi$ of length $n$ over the alphabet $[a]$ which do not start with the letter $a$ (including the empty word) according to the number of occurrences of $\tau$ in $\pi$. We then have

$$
G^{\prime}=G-x^{\ell-2}\left(G^{\prime \prime}-1\right)+x^{\ell-2} y\left(G^{\prime \prime}-1\right)
$$

and

$$
G=G^{\prime \prime}+x G^{\prime}
$$

from the definitions since words enumerated by $G^{\prime}$ and starting with $\ell-2$ a's followed by any letter $j$, where $j<a$, have an additional occurrence of $\tau$ at the beginning. Combining the two prior relations yields

$$
G^{\prime}=\frac{\left(1-x^{\ell-2}(1-y)\right) G+x^{\ell-2}(1-y)}{1-x^{\ell-1}(1-y)} .
$$

Since the patterns $\tau=22 \cdots 21$ and $\tau=11 \cdots 12$ are equivalent on words in $[k]$ (simply replace each letter $r$ with $k+1-r$ ), Theorem 2.2 in [1] implies

$$
G=\frac{x^{\ell-2}(y-1)}{1-x^{\ell-2}(1-y)-\left(1-x^{\ell-1}(1-y)\right)^{a}},
$$

which then yields

$$
G^{\prime}=\frac{x^{\ell-2}(y-1)\left(1-x^{\ell-1}(1-y)\right)^{a-1}}{1-x^{\ell-2}(1-y)-\left(1-x^{\ell-1}(1-y)\right)^{a}} .
$$


(This formula also holds when $a=1$.) From the fact that each partition $\pi$ of $[n]$ with exactly $k$ blocks may be expressed uniquely as $\pi=1 \pi^{(1)} 2 \pi^{(2)} \cdots k \pi^{(k)}$ such that each $\pi^{(i)}$ is a word over the alphabet $[i]$, we have that the generating function $F_{\tau}(x, y, k)$ is given by $x^{k} \prod_{a=1}^{k} G^{\prime}(x, y, a)$, which completes the proof.

Letting $\ell=3$ in Theorem 2.7 yields

$$
F_{221}(x, y, k)=x^{2 k}(y-1)^{k} \prod_{a=1}^{k} \frac{\left(1-x^{2}(1-y)\right)^{a-1}}{1-x(1-y)-\left(1-x^{2}(1-y)\right)^{a}}
$$

and letting $y=0$ in this implies that the generating function for the number of partitions of $[n]$ with $k$ parts which avoid the pattern 221 is given by

$$
F_{221}(x, 0, k)=x^{2 k} \prod_{a=1}^{k} \frac{\left(1-x^{2}\right)^{a-1}}{\left(1-x^{2}\right)^{a}+x-1} .
$$

From Theorem 2.7, we have

$$
F_{\tau}(x, 1, k)=\lim _{y \rightarrow 1} F_{\tau}(x, y, k)=x^{k} \prod_{j=1}^{k} \lim _{y \rightarrow 1}\left(\frac{f_{j}(y)}{g_{j}(y)}\right),
$$

where $f_{j}(y)=x^{\ell-2}(y-1)\left(1-x^{\ell-1}(1-y)\right)^{j-1}$ and $g_{j}(y)=1-x^{\ell-2}(1-y)-\left(1-x^{\ell-1}(1-y)\right)^{j}$. Since $f_{j}(1)=g_{j}(1)=0, f_{j}^{\prime}(1)=x^{\ell-2}$, and $g_{j}^{\prime}(1)=x^{\ell-2}-j x^{\ell-1}$, where primes denote differentiation with respect to $y$, we see that

$$
\lim _{y \rightarrow 1}\left(\frac{f_{j}(y)}{g_{j}(y)}\right)=\frac{1}{1-j x}
$$

and hence $F_{\tau}(x, y, k)$ reduces to the ordinary generating function for $S_{n, k}$ whenever $y=1$.

Theorem 2.7 also implies

$$
\left.\frac{d}{d y} F_{\tau}(x, y, k)\right|_{y=1}=F_{\tau}(x, 1, k) \sum_{j=1}^{k} \lim _{y \rightarrow 1}\left(\frac{f_{j}^{\prime}(y) g_{j}(y)-f_{j}(y) g_{j}^{\prime}(y)}{f_{j}(y) g_{j}(y)}\right) .
$$

Since $f_{j}^{\prime \prime}(1)=2(j-1) x^{2 \ell-3}$ and $g_{j}^{\prime \prime}(1)=-j(j-1) x^{2 \ell-2}$, two applications of L'Hôpital's rule implies

$$
\begin{aligned}
\lim _{y \rightarrow 1}\left(\frac{f_{j}^{\prime}(y) g_{j}(y)-f_{j}(y) g_{j}^{\prime}(y)}{f_{j}(y) g_{j}(y)}\right) & =\lim _{y \rightarrow 1}\left(\frac{f_{j}^{\prime \prime}(y) g_{j}^{\prime}(y)-f_{j}^{\prime}(y) g_{j}^{\prime \prime}(y)}{2 f_{j}^{\prime}(y) g_{j}^{\prime}(y)}\right) \\
& =\frac{f_{j}^{\prime \prime}(1)}{2 f_{j}^{\prime}(1)}-\frac{g_{j}^{\prime \prime}(1)}{2 g_{j}^{\prime}(1)}=(j-1) x^{\ell-1}+\frac{\left(\begin{array}{l}
j \\
2
\end{array}\right) x^{\ell}}{1-j x}
\end{aligned}
$$

so that

$$
\left.\frac{d}{d y} F_{\tau}(x, y, k)\right|_{y=1}=F_{\tau}(x, 1, k)\left(x^{\ell-1}\left(\begin{array}{l}
k \\
2
\end{array}\right)+\sum_{j=1}^{k} \frac{x^{\ell}\left(\begin{array}{l}
j \\
2
\end{array}\right)}{1-j x}\right) .
$$

This yields the following corollary to Theorem 2.7. 
Corollary 2.8. The total number of occurrences of the subword $22 \cdots 21 \in[2]^{\ell}$ in all of the partitions of $[n]$ with exactly $k$ blocks is given by

$$
\left(\begin{array}{l}
k \\
2
\end{array}\right) S_{n-\ell+1, k}+\sum_{j=2}^{k}\left(\begin{array}{l}
j \\
2
\end{array}\right) f_{n, j},
$$

where $f_{n, j}=\sum_{i=\ell}^{n-k} j^{i-\ell} S_{n-i, k}$ and $S_{i, j}$ is the Stirling number of the second kind.

We now provide a combinatorial proof of Corollary 2.8. For this, it is enough to show, by Lemma 2.5, that the total number of occurrences of $\tau=22 \cdots 21$ within the members of $P(n, k)$ in which the element of $[n]$ corresponding to the first 2 is minimal equals $\left(\begin{array}{c}k \\ 2\end{array}\right) S_{n-\ell+1, k}$. By prior reasoning, note that occurrences of $\tau=22 \cdots 21$ within members of $P(n, k)$ in which the first 2 is minimal are synonymous with occurrences of 21 within members of $P(n-\ell+2, k)$ in which the 2 is minimal. To complete the proof, we must then show that there are $\left(\begin{array}{l}k \\ 2\end{array}\right) S_{r-1, k}$ occurrences of 21 in which the 2 is minimal within the members of $P(r, k)$. To see this, first choose two numbers $a<b$ in $[k]$. Given $\lambda \in P(r-1, k)$, let $m$ denote the smallest member of block $b$. Increase all members of $[m+1, r-1]$ by one (leaving them within their blocks) and then add the element $m+1$ to block $a$. This produces an occurrence of 21 (in the form $b a$ ) at positions $m$ and $m+1$ within some member of $P(r, k)$.

\subsection{The subword pattern $\tau=m \rho m$}

Theorem 2.9. Let $\tau=m \rho m \in[m]^{\ell}$ be a subword pattern, where $\rho$ does not contain $m$. Then the generating function $F_{\tau}(x, y, k)$ is given by

$$
\frac{x^{k}}{(1-x)(1-2 x) \cdots(1-(m-1) x)} \prod_{a=m}^{k} \frac{\frac{1}{1+x^{\ell-1}\left(\begin{array}{c}
a-1 \\
m-1
\end{array}\right)(1-y)}}{1-(m-1) x-x \sum_{j=m-1}^{a-1} \frac{1}{1+\left(\begin{array}{c}
j \\
m-1
\end{array}\right) x^{\ell-1}(1-y)}} .
$$

Proof. Let $G_{a}(x, y)$ be the generating function for the number of words $\pi$ of length $n$ over the alphabet $[a]$ according to the number of occurrences of the subword pattern $\tau$ in $a \pi(a+1)$. Since $\pi$ either contains the letter $a$ (here we write $\pi=\pi^{\prime} a \pi^{\prime \prime}$ where $\pi^{\prime}$ is a word which does not contain $a$ ) or does not, we see that

$$
G_{a}(x, y)=G_{a-1}^{\prime}(x, y)+x\left(G_{a-1}^{\prime}(x, y)-x^{\ell-2}\left(\begin{array}{c}
a-1 \\
m-1
\end{array}\right)(1-y)\right) G_{a}(x, y)
$$

which is equivalent to

$$
G_{a}(x, y)=\frac{G_{a-1}^{\prime}(x, y)}{1-x\left(G_{a-1}^{\prime}(x, y)-x^{\ell-2}\left(\begin{array}{c}
a-1 \\
m-1
\end{array}\right)(1-y)\right)},
$$


where $G_{a}^{\prime}(x, y)$ is the generating function for the number of words of length $n$ over the alphabet $[a]$ according to the number occurrences of the subword pattern $\tau$. From $[1$, Theorem 2.7], we have that

$$
G_{a}^{\prime}(x, y)=\frac{1}{1-\min \{m-1, a\} x-x \sum_{j=m-1}^{a-1} \frac{1}{1+\left(\begin{array}{c}
j \\
m-1
\end{array}\right) x^{\ell-1}(1-y)}}, \quad a \geqslant 1 .
$$

From the fact that each partition $\pi$ of $[n]$ with exactly $k$ blocks may be expressed uniquely as $\pi=1 \pi^{(1)} 2 \pi^{(2)} \cdots k \pi^{(k)}$ such that $\pi^{(i)}$ is a word over the alphabet $[i]$, we have that the generating function $F_{\tau}(x, y, k)$ is given by $x^{k} \prod_{a=1}^{k} G_{a}(x, y)$. Hence, by (2) and (3), we get the following expression for $G_{a}(x, y)$ :

$$
\frac{1}{\left(1+x^{\ell-1}\left(\begin{array}{c}
a-1 \\
m-1
\end{array}\right)(1-y)\right)\left(1-\min \{m-1, a-1\} x-x \sum_{j=m-1}^{a-2} \frac{1}{1+\left(\begin{array}{c}
j \\
m-1
\end{array}\right) x^{\ell-1}(1-y)}\right)-x},
$$

which implies our theorem.

When $y=1$ in Theorem 2.9, the generating function reduces to that of the Stirling number of the second kind. Taking $\tau=211 \cdots 12 \in[2]^{\ell}$ in Theorem 2.9 gives

$$
F_{\tau}(x, y, k)=x^{k} \prod_{a=1}^{k} \frac{\frac{1}{1+x^{\ell-1}(a-1)(1-y)}}{1-x-x \sum_{j=1}^{a-1} \frac{1}{1+j x^{\ell-1}(1-y)}} .
$$

In particular, when $\ell=3$ and $y=0$, we see that the generating function for the number of partitions of $[n]$ with exactly $k$ blocks that avoid the subword pattern $\tau=212$ is given by

$$
F_{212}(x, 0, k)=\frac{x^{k}}{1-x} \prod_{a=1}^{k-1} \frac{\frac{1}{1+a x^{2}}}{1-x \sum_{j=0}^{a} \frac{1}{1+j x^{2}}} .
$$

Differentiating the generating function in Theorem 2.9 gives

$$
\begin{aligned}
\left.\frac{d}{d y} F_{\tau}(x, y, k)\right|_{y=1} & =F_{\tau}(x, 1, k)\left(\sum_{a=m}^{k}\left[x^{\ell-1}\left(\begin{array}{c}
a-1 \\
m-1
\end{array}\right)+\frac{1}{1-a x} \sum_{j=1}^{a-1} x^{\ell}\left(\begin{array}{c}
j \\
m-1
\end{array}\right)\right]\right) \\
& =F_{\tau}(x, 1, k)\left(x^{\ell-1}\left(\begin{array}{c}
k \\
m
\end{array}\right)+\sum_{a=1}^{k} \frac{x^{\ell}\left(\begin{array}{c}
a \\
m
\end{array}\right)}{1-a x}\right),
\end{aligned}
$$

which yields the following corollary.

Corollary 2.10. The total number of occurrences of the subword $\tau=m \rho m \in[m]^{\ell}$, where $\rho$ does not contain $m$, in all of the partitions of $[n]$ with exactly $k$ blocks is given by

$$
\left(\begin{array}{c}
k \\
m
\end{array}\right) S_{n-\ell+1, k}+\sum_{j=m}^{k}\left(\begin{array}{c}
j \\
m
\end{array}\right) f_{n, j}
$$

where $f_{n, j}=\sum_{i=\ell}^{n-k} j^{i-\ell} S_{n-i, k}$ and $S_{i, j}$ is the Stirling number of the second kind. 
We now provide a combinatorial explanation of Corollary 2.10. By Lemma 2.5, the second term of the explicit formula counts all primary occurrences of the subword pattern $\tau$ within all members of $P(n, k)$. To complete the proof, we must then show that there are $\left(\begin{array}{c}k \\ m\end{array}\right) S_{n-\ell+1, k}$ total occurrences of $\tau$ which aren't primary (which we'll term non-primary). Note that the first letter of a non-primary occurrence of $\tau$ in a partition $\lambda$ must correspond to a minimal element of $\pi$, with all the other letters comprising the occurrence nonminimal. Then given $m$ numbers $a_{1}<a_{2}<\cdots<a_{m}$ in $[k]$ and $\lambda \in P(n-\ell+1, k)$, let $t$ denote the smallest member of block $a_{m}$. Increase all members of $[t+1, n-\ell+1]$ by $\ell-1$ in $\lambda$ (leaving them within their current blocks). Then add the element $t+i$ to block $a_{r}$, where $r$ denotes the $(i+1)^{s t}$ letter of the subword $\tau$ for all $i, 1 \leqslant i \leqslant \ell-1$. The resulting member of $P(n, k)$ will have a non-primary occurrence of $\tau$ starting at the $t^{\text {th }}$ letter.

\subsection{The subword pattern $\tau=m \rho(m+1)$}

Theorem 2.11. Let $\tau=m \rho(m+1) \in[m+1]^{\ell}$ be a subword pattern, where $\rho$ does not contain $m$ and $m+1$. Then the generating function $F_{\tau}(x, y, k)$ with $k \geqslant m+1$ is given by

$$
\frac{x^{k} B_{k}(x, y)}{(1-x) \cdots(1-(m-1) x)} \prod_{a=m}^{k-1} \frac{A_{a}(x, y)-\left(\begin{array}{c}
a-1 \\
m-1
\end{array}\right) x^{\ell-2}(1-y)}{1-x A_{a}(x, y)}
$$

where

$$
\begin{aligned}
B_{a}(x, y) & =\frac{1}{1-(m-1) x-x \sum_{i=m-2}^{a-2} \prod_{j=m-2}^{i}\left(1-\left(_{m-1}^{j}\right) x^{\ell-1}(1-y)\right)}, \quad a \geqslant m, \\
A_{a}(x, y) & =\frac{\prod_{j=m-1}^{a-2}\left(1-\left(\begin{array}{c}
j \\
m-1
\end{array}\right) x^{\ell-1}(1-y)\right)}{1-m x-x \sum_{i=m-1}^{a-3} \prod_{j=m-1}^{i}\left(1-\left(_{m-1}^{j}\right) x^{\ell-1}(1-y)\right)}, \quad a \geqslant m+1,
\end{aligned}
$$

and $A_{m}(x, y)=\frac{1}{1-(m-1) x}$.

Proof. Let $A_{a}=A_{a}(x, y)$ be the generating function for the number of words $\pi$ of length $n$ over the alphabet $[a-1]$ according to the number occurrences of the subword pattern $\tau$ in $\pi a$. Let $A_{a}^{\prime}=A_{a}^{\prime}(x, y)$ be the generating function for the number of words $\pi$ of length $n$ over the alphabet $[a-1]$ according to the number of occurrences of the subword pattern $\tau$ in $a \pi(a+1)$. Clearly, $A_{a}^{\prime}=A_{a}-\left(\begin{array}{c}a-1 \\ m-1\end{array}\right) x^{\ell-2}(1-y)$.

Note that each word $\pi$ over the alphabet $[a-1]$ either does not contain $a-1$ or may be expressed as $\pi^{(1)}(a-1) \pi^{(2)}(a-1) \cdots \pi^{(s)}(a-1) \pi^{(s+1)}$ such that $\pi^{(j)}$ is a word over the alphabet $[a-2]$, for all $j$. Therefore

$$
A_{a}=A_{a-1}+\frac{x A_{a-1} A_{a-1}^{\prime}}{1-x A_{a-1}}
$$

which implies that

$$
A_{a}=A_{a-1}+\frac{x A_{a-1}\left(A_{a-1}-\left(\begin{array}{c}
a-2 \\
m-1
\end{array}\right) x^{\ell-2}(1-y)\right)}{1-x A_{a-1}}=\frac{A_{a-1}\left(1-\left(\begin{array}{c}
a-2 \\
m-1
\end{array}\right) x^{\ell-1}(1-y)\right)}{1-x A_{a-1}} .
$$


Hence, by induction on $a$, we have $A_{a}=\frac{1}{1-(a-1) x}$ for all $a=1,2, \ldots, m$, and

$$
A_{a}(x, y)=\frac{\prod_{j=m-1}^{a-2}\left(1-\left(_{m-1}^{j}\right) x^{\ell-1}(1-y)\right)}{1-m x-x \sum_{i=m-1}^{a-3} \prod_{j=m-1}^{i}\left(1-\left(\begin{array}{c}
j \\
m-1
\end{array}\right) x^{\ell-1}(1-y)\right)},
$$

for all $a \geqslant m+1$.

Let $B_{a}=B_{a}(x, y)$ be the generating function for the number of words $\pi$ of length $n$ over the alphabet $[a]$ according to the number of occurrences of the subword pattern $\tau$ in $\pi$. From [1, Theorem 2.11], we can state that

$$
B_{a}=\frac{1}{1-(m-1) x-x \sum_{i=m-2}^{a-2} \prod_{j=m-2}^{i}\left(1-\left(\begin{array}{c}
j \\
m-1
\end{array}\right) x^{\ell-1}(1-y)\right)}, \quad a \geqslant m .
$$

Hence, the generating function for the number words $\pi$ of length $n$ over the alphabet $[a]$ according to the number of occurrences of the subword pattern $\tau$ in $a \pi(a+1)$ (decompose the word $\pi$ as $\pi^{(1)} a \pi^{(2)} a \cdots \pi^{(s)} a \pi^{(s+1)}$, where $\pi^{(j)}$ is a word over the alphabet $\left.[a-1]\right)$ is given by

$$
\frac{A_{a}^{\prime}}{1-x A_{a}}=\frac{A_{a}-\left(\begin{array}{c}
a-1 \\
m-1
\end{array}\right) x^{\ell-2}(1-y)}{1-x A_{a}}, \quad a \geqslant m
$$

and $\frac{1}{1-a x}$ for $a=1,2, \ldots, m-1$. From the fact that each partition $\pi$ of $[n]$ with exactly $k$ blocks can be written as $\pi=1 \pi^{(1)} 2 \pi^{(2)} \cdots k \pi^{(k)}$ such that $\pi^{(i)}$ is a word over the alphabet $[i]$, we have that the generating function $F_{\tau}(x, y, k)$ is given by

$$
\frac{x^{k} B_{k}}{(1-x) \cdots(1-(m-1) x)} \prod_{a=m}^{k-1} \frac{A_{a}-\left(\begin{array}{c}
a-1 \\
m-1
\end{array}\right) x^{\ell-2}(1-y)}{1-x A_{a}}
$$

which completes the proof.

For instance, Theorem 2.11 for $\tau=213$ implies that the generating function for the number of partitions of $[n]$ with exactly $k$ blocks according to the number occurrences of the subword pattern 213 is given by

$$
F_{213}(x, y, k)=\frac{x^{k} \prod_{a=2}^{k-1} \frac{A_{a}-(a-1) x(1-y)}{1-x A_{a}}}{(1-x)\left[1-x-x \sum_{i=0}^{k-2} \prod_{j=0}^{i}\left(1-j x^{2}(1-y)\right)\right]}, \quad k \geqslant 2,
$$

where

$$
A_{a}=\frac{\prod_{j=1}^{a-2}\left(1-j x^{2}(1-y)\right)}{1-2 x-x \sum_{i=1}^{a-3} \prod_{j=1}^{i}\left(1-j x^{2}(1-y)\right)} .
$$

This implies that $F_{213}(x, 1, k)=\frac{x^{k}}{(1-x)(1-2 x) \cdots(1-k x)}$, as it is well known. One may readily verify that

$$
A_{a}(1):=\left.A_{a}(x, y)\right|_{y=1}=\frac{1}{1-(a-1) x}
$$


and

$$
d A_{a}(1):=\left.\frac{d}{d y} A_{a}(x, y)\right|_{y=1}=\frac{x^{\ell-1}\left(\begin{array}{c}
a-1 \\
m
\end{array}\right)}{1-(a-1) x}+\frac{x^{\ell}\left(\begin{array}{c}
a-1 \\
m+1
\end{array}\right)}{[1-(a-1) x]^{2}} .
$$

Combining these facts with Theorem 2.11 and the fact that

$$
\left.\frac{d}{d y} B_{k}(x, y)\right|_{y=1}=\frac{x^{\ell}}{1-k x} \sum_{i=m-2}^{k-2}\left(\begin{array}{c}
i+1 \\
m
\end{array}\right)=\frac{x^{\ell}\left(\begin{array}{c}
k \\
m+1
\end{array}\right)}{1-k x}
$$

yields

$$
\begin{aligned}
& \left.\frac{d}{d y} F_{\tau}(x, y, k)\right|_{y=1} \\
& =F_{\tau}(x, 1, k)\left(\sum_{a=m}^{k-1}\left[d A_{a}(1)+\left(\begin{array}{c}
a-1 \\
m-1
\end{array}\right) x^{\ell-2}\right][1-(a-1) x]+\sum_{a=m}^{k-1} \frac{x d A_{a}(1)}{1-x A_{a}(1)}+\frac{x^{\ell}\left(\begin{array}{c}
k \\
m+1
\end{array}\right)}{1-k x}\right) \\
& =F_{\tau}(x, 1, k)\left(\sum_{a=m}^{k-1}\left(x^{\ell-2}\left(\begin{array}{c}
a-1 \\
m-1
\end{array}\right)-(m-1) x^{\ell-1}\left(\begin{array}{c}
a \\
m
\end{array}\right)\right)+\sum_{a=m}^{k} \frac{x^{\ell}\left(\begin{array}{c}
a \\
m+1
\end{array}\right)}{1-a x}\right) \\
& =F_{\tau}(x, 1, k)\left(x^{\ell-2}\left(\begin{array}{c}
k-1 \\
m
\end{array}\right)-x^{\ell-1}(m-1)\left(\begin{array}{c}
k \\
m+1
\end{array}\right)+\sum_{a=m}^{k} \frac{x^{\ell}\left(\begin{array}{c}
a \\
m+1
\end{array}\right)}{1-a x}\right),
\end{aligned}
$$

which implies the following corollary.

Corollary 2.12. The total number of occurrences of the subword $\tau=m \rho(m+1) \in[m+1]^{\ell}$, where $\rho$ does not contain $m$ or $m+1$, in all of the partitions of $[n]$ with exactly $k$ blocks is given by

$$
\left(\begin{array}{c}
k-1 \\
m
\end{array}\right) S_{n-\ell+2, k}-(m-1)\left(\begin{array}{c}
k \\
m+1
\end{array}\right) S_{n-\ell+1, k}+\sum_{j=m+1}^{k}\left(\begin{array}{c}
j \\
m+1
\end{array}\right) f_{n, j}
$$

where $f_{n, j}=\sum_{i=\ell}^{n-k} j^{i-\ell} S_{n-i, k}$ and $S_{i, j}$ is the Stirling number of the second kind.

We now provide a combinatorial proof of Corollary 2.12. By Lemma 2.5, to complete the proof, we must show that the total number of non-primary occurrences of $\tau=m \rho(m+$ $1)$ is given by $\left(\begin{array}{c}k-1 \\ m\end{array}\right) S_{n-\ell+2, k}-(m-1)\left(\begin{array}{c}k \\ m+1\end{array}\right) S_{n-\ell+1, k}$, which we rewrite as $2\left(\begin{array}{c}k \\ m+1\end{array}\right) S_{n-\ell+1, k}+$ $\left(\begin{array}{c}k-1 \\ m\end{array}\right) S_{n-\ell+1, k-1}$, using the facts that $S_{n-\ell+2, k}=k S_{n-\ell+1, k}+S_{n-\ell+1, k-1}$ and $k\left(\begin{array}{c}k-1 \\ m\end{array}\right)=$ $(m+1)\left(\begin{array}{c}k \\ m+1\end{array}\right)$. First observe that if an occurrence of $\tau=m \rho(m+1)$ is non-primary within a partition $\pi$, then the number corresponding to the slot containing $m+1$ must be a minimal element of $\pi$ (for if not, then the number corresponding to the slot containing $m$ would not be minimal as well, which would imply that the occurrence of $\tau$ within $\pi$ is primary, a contradiction). There are then $\left(\begin{array}{c}k \\ m+1\end{array}\right) S_{n-\ell+1, k}$ non-primary occurrences of $\tau$ in which the number corresponding to the slot containing $m$ is not minimal. To see 
this, first pick $m+1$ numbers $a_{1}<a_{2}<\cdots<a_{m+1}$ in $[k]$ and let $t$ denote the smallest element of block $a_{m+1}$ in $\lambda \in P(n-\ell+1, k)$. Increase all members of $[t, n-\ell+1]$ by $\ell-1$ within $\lambda$, leaving all members within their current blocks. Then add the element $t+i$ to block $a_{r}$, where $r$ denotes the $(i+1)^{s t}$ letter of the subword pattern $\tau$ for all $i$, $0 \leqslant i \leqslant \ell-2$. Since the minimal elements of the blocks $a_{i}$ for $1 \leqslant i \leqslant m$ are all less than $t$, the resulting member of $P(n, k)$ will have a non-primary occurrence of $\tau$ starting at $t$ in which the number corresponding to $m$ (namely, $t$ ) is not minimal.

By similar reasoning, there are $\left(\begin{array}{c}k \\ m+1\end{array}\right) S_{n-\ell+1}$ non-primary occurrences of $\tau$ in which the number corresponding to the slot containing $m$ is minimal but does not occur as a singleton block. To see this, pick $m+1$ numbers $a_{1}<a_{2}<\cdots<a_{m+1}$ in $[k]$ and let $t$ denote the smallest element of block $a_{m}$ of $\lambda \in P(n-\ell+1, k)$. Increase all members of $[t, n-\ell+1]$ by $\ell-1$ within $\lambda$ and add $t$ to block $a_{m+1}$. Then add $t+i$ to block $a_{r}$, where $r$ denotes the $(i+1)^{s t}$ letter of $\tau, 1 \leqslant i \leqslant \ell-2$. The resulting member of $P(n, k)$ will have a non-primary occurrence of $\tau$ starting at $t$ in which the letter corresponding to $m$ (namely, $t$ ) is minimal but does not occur as a singleton block. Finally, there are $\left(\begin{array}{c}k-1 \\ m\end{array}\right) S_{n-\ell+1, k-1}$ non-primary occurrences of $\tau$ in which the letter corresponding to $m$ occurs as a singleton block. To see this, pick $a_{1}<a_{2}<\cdots<a_{m}$ in $[k-1]$ and let $t$ denote the smallest element of block $a_{m}$ of $\lambda \in P(n-\ell+1, k-1)$. Increase all members of $[t, n-\ell+1]$ by $\ell-1$ within $\lambda$ and add the singleton block $\{t\}$. Then add $t+i$ to block $a_{r}$, where $r$ denotes the $(i+1)^{s t}$ letter of the subword $\tau, 1 \leqslant i \leqslant \ell-2$. The resulting member of $P(n, k)$ will have a non-primary occurrence of $\tau$ starting at $t$ in which the singleton block $\{t\}$ occurs.

\subsection{The subword pattern $\tau=(m+1) \rho m$}

Theorem 2.13. Let $\tau=(m+1) \rho m \in[m+1]^{\ell}$ be a subword pattern, where $\rho$ does not contain $m$ and $m+1$. Then the generating function $F_{\tau}(x, y, k)$ with $k \geqslant 1$ is given by

$$
x^{k} \prod_{a=1}^{k} \frac{A_{a}(x, y)}{1-x A_{a}(x, y)}
$$

where

$$
A_{a}(x, y)=\frac{\prod_{j=m-1}^{a-2}\left(1-\left(_{m-1}^{j}\right) x^{\ell-1}(1-y)\right)}{1-m x-x \sum_{i=m-1}^{a-3} \prod_{j=m-1}^{i}\left(1-\left(_{m-1}^{j}\right) x^{\ell-1}(1-y)\right)}, \quad a \geqslant m+1,
$$

and $A_{a}(x, y)=\frac{1}{1-(a-1) x}$ for $a \leqslant m$.

Proof. Let $C_{a}=C_{a}(x, y)$ be the generating function for the number of words $\pi$ of length $n$ over the alphabet $[a]$ according to the number of occurrences of the subword pattern $\tau$ in $a \pi$. Since each word $\pi$ over the alphabet $[a]$ either does not contain the letter $a$ or may be written as $\pi^{\prime} a \pi^{\prime \prime}$, where $\pi^{\prime}$ is a word over the alphabet $[a-1]$ and $\pi^{\prime \prime}$ is a word over the alphabet $[a]$, we have

$$
C_{a}=C_{a}^{\prime}+x C_{a}^{\prime} C_{a}
$$


where $C_{a}^{\prime}$ is the generating function for the number of words $\pi$ of length $n$ over the alphabet $[a-1]$ according to the number of occurrences of the subword pattern $\tau$ in $a \pi$. From the proof of Theorem 2.11 and the reversal operation (map each word $\pi_{1} \cdots \pi_{n}$ to $\pi_{n} \cdots \pi_{1}$ ), we have $C_{a}^{\prime}=A_{a}$. Hence

$$
C_{a}=\frac{A_{a}}{1-x A_{a}}
$$

From the fact that each partition $\pi$ of $[n]$ with exactly $k$ blocks may be expressed uniquely as $\pi=1 \pi^{(1)} 2 \pi^{(2)} \cdots k \pi^{(k)}$ such that each $\pi^{(i)}$ is a word over the alphabet $[i]$, we have that the generating function $F_{\tau}(x, y, k)$ is given by $x^{k} \prod_{a=1}^{k} \frac{A_{a}}{1-x A_{a}}$, which completes the proof.

By way of example, Theorem 2.13 for $\tau=312$ implies that the generating function $F_{312}(x, y, k)$ for the number of partitions of $[n]$ with exactly $k$ blocks according to the number of occurrences of the subword pattern 312 is given by

$$
\frac{x^{k} \prod_{j=1}^{k-2}\left(1-j x^{2}(1-y)\right)^{k-1-j}}{(1-x)(1-2 x) \prod_{a=1}^{k-2}\left(1-2 x-x \sum_{i=1}^{a} \prod_{j=1}^{i}\left(1-j x^{2}(1-y)\right)\right)} .
$$

From (4) and (5), we have from Theorem 2.13 that

$$
\begin{aligned}
& \left.\frac{d}{d y} F_{\tau}(x, y, k)\right|_{y=1}=F_{\tau}(x, 1, k) \sum_{a=m+1}^{k}\left(\frac{d A_{a}(1)}{A_{a}(1)}+\frac{x d A_{a}(1)}{1-x A_{a}(1)}\right) \\
& =F_{\tau}(x, 1, k) \sum_{a=m+1}^{k}\left(x^{\ell-1}\left(\begin{array}{c}
a-1 \\
m
\end{array}\right)+\frac{x^{\ell}\left(\begin{array}{c}
a-1 \\
m+1
\end{array}\right)}{1-(a-1) x}+\frac{x^{\ell}\left(\begin{array}{c}
a-1 \\
m
\end{array}\right)}{1-a x}+\frac{x^{\ell+1}\left(\begin{array}{c}
a-1 \\
m+1
\end{array}\right)}{(1-a x)(1-(a-1) x)}\right) \\
& =F_{\tau}(x, 1, k)\left[x^{\ell-1}\left(\begin{array}{c}
k \\
m+1
\end{array}\right)+\sum_{a=m+1}^{k} \frac{x^{\ell}\left(\begin{array}{c}
a \\
m+1
\end{array}\right)}{1-a x}\right],
\end{aligned}
$$

which implies the following corollary.

Corollary 2.14. The total number of occurrences of the subword $\tau=(m+1) \rho m \in[m+1]^{\ell}$, where $\rho$ does not contain $m$ or $m+1$, in all of the partitions of $[n]$ with exactly $k$ blocks is given by

$$
\left(\begin{array}{c}
k \\
m+1
\end{array}\right) S_{n-\ell+1, k}+\sum_{j=m+1}^{k}\left(\begin{array}{c}
j \\
m+1
\end{array}\right) f_{n, j},
$$

where $f_{n, j}=\sum_{i=\ell}^{n-k} j^{i-\ell} S_{n-i, k}$ and $S_{i, j}$ is the Stirling number of the second kind.

We remark that a combinatorial proof may be given for Corollary 2.14 similar to that given above for Corollary 2.10. 


\section{Three letter subwords}

In this section, we turn our attention to counting occurrences of three letter subword patterns within members of $P(n . k)$. Theorem 2.1 of [8] implies

$$
F_{111}(x, y, k)=\frac{\left(x+x^{2}+x^{3} /(1-x y)\right)^{k}}{\prod_{j=0}^{k-1}\left(1-j\left(x+x^{2}+x^{3} /(1-x y)\right)\right)} .
$$

The expressions for $F_{123}(x, y, k)$ and $F_{321}(x, y, k)$ are more complicated and occur as Theorem 3.6 and Corollary 4.4 of [8]. From our results in previous sections, we have the following:

$$
\begin{aligned}
& F_{112}(x, y, k)=F_{122}(x, y, k)=x^{k} \frac{(1-x(1-y))^{k-1}}{\prod_{a=1}^{k}\left(1-x \sum_{j=0}^{a-1}\left(1-x^{2}(1-y)\right)^{j}\right)}, \\
& F_{211}(x, y, k)=F_{221}(x, y, k)=x^{2 k}(y-1)^{k} \prod_{a=1}^{k} \frac{\left(1-x^{2}(1-y)\right)^{a-1}}{1-x(1-y)-\left(1-x^{2}(1-y)\right)^{a}}, \\
& F_{212}(x, y, k)=x^{k} \prod_{a=1}^{k} \frac{\frac{1}{1+(a-1) x^{2}(1-y)}}{1-x-x \sum_{j=1}^{a-1} \frac{1}{1+j x^{2}(1-y)}}, \\
& F_{213}(x, y, k)=\frac{x^{k} \prod_{a=2}^{k-1} \frac{A_{a}-(a-1) x(1-y)}{1-x A_{a}}}{(1-x)\left[1-x-x \sum_{i=0}^{k-2} \prod_{j=0}^{i}\left(1-j x^{2}(1-y)\right)\right]}, \quad k \geqslant 2, \\
& F_{312}(x, y, k)=\frac{x^{k} \prod_{j=1}^{k-2}\left(1-j x^{2}(1-y)\right)^{k-1-j}}{(1-x)(1-2 x) \prod_{a=1}^{k-2}\left(1-2 x-x \sum_{i=1}^{a} \prod_{j=1}^{i}\left(1-j x^{2}(1-y)\right)\right)},
\end{aligned}
$$

where

$$
A_{a}=\frac{\prod_{j=1}^{a-2}\left(1-j x^{2}(1-y)\right)}{1-2 x-x \sum_{i=1}^{a-3} \prod_{j=1}^{i}\left(1-j x^{2}(1-y)\right)} .
$$

The remaining cases, namely 121,132 , and 231 , seem to be more difficult. We will use more advanced algebraic techniques to derive both recurrences of the generating function for these patterns as well as find an explicit formula of the generating function for the total number of occurrences of these patterns.

Theorem 3.1. Let $G_{k}=G_{k}(x, y)=F_{121}(x, y, k)$. Then the generating function $G_{k}$ satisfies the recurrence relation

$$
\begin{aligned}
G_{k}=x \sum_{a=1}^{k-1} \sum_{j=0}^{k-1-a} & \frac{x^{2 j}(y-1)^{j}}{\prod_{i=0}^{j}\left(1-(k-a-i) x^{2}(y-1)\right)} G_{k-j} \\
& +\sum_{a=1}^{k} \frac{x^{2(k-a)+1}(y-1)^{k-a}}{\prod_{i=0}^{k-1-a}\left(1-(k-a-i) x^{2}(y-1)\right)}\left(G_{a}+G_{a-1}\right)
\end{aligned}
$$

with the initial condition $G_{1}=\frac{x}{1-x}$. 
Proof. Let $G_{k}\left(a_{s} \cdots a_{1}\right)=G_{k}\left(x, y \mid a_{s} \cdots a_{1}\right)$ be the generating function for the number of partitions $\pi=\pi_{1} \cdots \pi_{n}, \pi_{n+1-j}=a_{j}$ for all $j=1,2, \ldots, s$ with exactly $k$ blocks according to the number of occurrences of the subword pattern 121. Define $G_{k}=G_{k}(x, y)=$ $F_{121}(x, y, k)$. From the definitions, we have

$$
\begin{aligned}
G_{k}(k) & =x G_{k}+x G_{k-1}, \\
G_{k}(a) & =\sum_{j=1}^{k} G_{k}(j a)=x \sum_{j=1}^{a} G_{k}(j)+\sum_{j=a+1}^{k} G_{k}(j a), \quad 1 \leqslant a \leqslant k-1, \\
G_{k}(j a) & =\sum_{i=1, i \neq a}^{j} G_{k}(i j a)+G_{k}(a j a)+\sum_{i=j+1}^{k} G_{k}(i j a) \\
& =x^{2} \sum_{i=1}^{j} G_{k}(i)+x^{2}(y-1) G_{k}(a)+x \sum_{i=j+1}^{k} G_{k}(i j), \quad a+1 \leqslant j \leqslant k-1, \\
G_{k}(k a) & =x^{2}\left(G_{k}-G_{k}(a)\right)+x^{2} y G_{k}(a)+x^{2}\left(G_{k-1}-G_{k-1}(a)\right)+x^{2} y G_{k-1}(a) \\
& =x^{2} G_{k}+x^{2}(y-1) G_{k}(a)+x^{2} G_{k-1}+x^{2}(y-1) G_{k-1}(a),
\end{aligned}
$$

which is equivalent to

$$
\begin{aligned}
G_{k}(k) & =x G_{k}+x G_{k-1}, \\
G_{k}(a) & =\sum_{j=1}^{k} G_{k}(j a)=x \sum_{j=1}^{a} G_{k}(j)+\sum_{j=a+1}^{k} G_{k}(j a), \quad 1 \leqslant a \leqslant k-1, \\
G_{k}(j a) & =x^{2} \sum_{i=1}^{j} G_{k}(i)+x^{2}(y-1) G_{k}(a)+x\left(G_{k}(j)-x \sum_{i=1}^{j} G_{k}(i)\right) \\
& =x G_{k}(j)+x^{2}(y-1) G_{k}(a), \\
G_{k}(k a) & =x^{2} G_{k}+x^{2}(y-1) G_{k}(a)+x^{2} G_{k-1}+x^{2}(y-1) G_{k-1}(a) .
\end{aligned}
$$

Thus, for all $a=1,2, \ldots, k-1$,

$$
\begin{aligned}
G_{k}(a)= & x \sum_{j=1}^{a} G_{k}(j)+\sum_{j=a+1}^{k} G_{k}(j a) \\
= & x \sum_{j=1}^{a} G_{k}(j)+\sum_{j=a+1}^{k-1}\left(x G_{k}(j)+x^{2}(y-1) G_{k}(a)\right) \\
& +x^{2} G_{k}+x^{2}(y-1) G_{k}(a)+x^{2} G_{k-1}+x^{2}(y-1) G_{k-1}(a) \\
= & x \sum_{j=1}^{k} G_{k}(j)+(k-a) x^{2}(y-1) G_{k}(a)+x^{2}(y-1) G_{k-1}(a) \\
= & x G_{k}+(k-a) x^{2}(y-1) G_{k}(a)+x^{2}(y-1) G_{k-1}(a),
\end{aligned}
$$


with the initial condition $G_{k}(k)=x G_{k}+x G_{k-1}$. Then the generating function $G_{k}(a)$ satisfies the recurrence relation

$$
G_{k}(a)=\frac{x}{1-(k-a) x^{2}(y-1)} G_{k}+\frac{x^{2}(y-1)}{1-(k-a) x^{2}(y-1)} G_{k-1}(a) .
$$

Iterating the above recurrence relation, we have

$$
\begin{aligned}
G_{k}(a)=x \sum_{j=0}^{k-1-a} \frac{x^{2 j}(y-1)^{j}}{\prod_{i=0}^{j}\left(1-(k-a-i) x^{2}(y-1)\right)} G_{k-j} \\
\quad+\frac{x^{2(k-a)+1}(y-1)^{k-a}}{\prod_{i=0}^{k-1-a}\left(1-(k-a-i) x^{2}(y-1)\right)}\left(G_{a}+G_{a-1}\right),
\end{aligned}
$$

for all $a=1,2, \ldots, k$. Summing over all possible values of $a$, we get the requested result.

Corollary 3.2. The generating function for the total number of occurrences of the subword pattern 121 in all the partitions of $[n]$ with exactly $k$ blocks is given by

$$
\frac{x^{k+1}}{(1-x)(1-2 x) \cdots(1-k x)} \sum_{j=2}^{k} \frac{\left(\begin{array}{l}
j \\
2
\end{array}\right) x^{2}+1-j x}{1-j x} .
$$

Proof. Theorem 3.1 for $y=1$ gives $G_{1}(x, 1)=\frac{x}{1-x}$ and

$$
G_{k}(x, 1)=(k-1) x G_{k}(x, 1)+x\left(G_{k}(x, 1)+G_{k-1}(x, 1)\right),
$$

which is equivalent to

$$
G_{k}(x, 1)=\frac{x^{k}}{\prod_{j=1}^{k}(1-j x)},
$$

as it is well known.

Define $G_{k}^{\prime}(x)=\left.\frac{d}{d y} G_{k}(x, y)\right|_{y=1}$. Theorem 3.1 gives

$$
\begin{aligned}
G_{k}^{\prime}(x)= & x \sum_{a=1}^{k-1}\left((k-a) x^{2} G_{k}(x, 1)+G_{k}^{\prime}(x)\right)+x \sum_{a=1}^{k-2} x^{2} G_{k-1}(x, 1) \\
& +x\left(G_{k}^{\prime}(x)+G_{k-1}^{\prime}(x)\right)+x^{3}\left(G_{k-1}(x, 1)+G_{k-2}(x, 1)\right),
\end{aligned}
$$

which is equivalent to

$$
G_{k}^{\prime}(x)=\frac{x}{1-k x} G_{k-1}^{\prime}(x)+\frac{x^{3}\left(\begin{array}{l}
k \\
2
\end{array}\right) G_{k}(x, 1)+x^{3}(k-1) G_{k-1}(x, 1)+x^{3} G_{k-2}(x, 1)}{1-k x} .
$$

Using

$$
G_{k}(x, 1)=\frac{x^{k}}{\prod_{j=1}^{k}(1-j x)},
$$

noting the initial condition $G_{1}^{\prime}(x)=0$, and iterating the above recurrence relation, we obtain the requested result. 
This yields the following explicit formula for the total number of occurrences of 121 .

Corollary 3.3. The total number of occurrences of the subword pattern 121 in all of the partitions of $[n]$ with exactly $k$ blocks is given by

$$
(k-1) S_{n-1, k}+\sum_{j=2}^{k}\left(\begin{array}{l}
j \\
2
\end{array}\right) f_{n, j}
$$

where $f_{n, j}=\sum_{i=3}^{n-k} j^{i-3} S_{n-i, k}$.

An argument similar to the one used in the above proof of Theorem 3.1 yields the following result.

Theorem 3.4. Let $G_{k}(a)=F_{132}(x, y \mid a)$ be the generating function for the number of partitions $\pi=\pi_{1} \cdots \pi_{n-1}$ a with exactly $k$ blocks according to the number occurrences of the subword pattern 132, and let $G_{k}=\sum_{a=1}^{k} G_{k}(a)=F_{132}(x, y, k)$. Then the generating function $G_{k}(a)$ satisfies the recurrence relation

$$
G_{k}(a)=x G_{k}+x^{2}(y-1)(k-a) \sum_{j=1}^{a-1} G_{k}(j)+x^{2}(y-1) \sum_{j=1}^{a-1} G_{k-1}(j)
$$

with the initial conditions $G_{k}(k)=x G_{k}+x G_{k-1}, G_{k}(1)=x G_{k}, G_{1}=G_{1}(1)=\frac{x}{1-x}$, $G_{2}(1)=\frac{x^{3}}{(1-x)(1-2 x)}, G_{2}(2)=\frac{x^{2}}{1-2 x}$ and $G_{2}=\frac{x^{2}}{(1-x)(1-2 x)}$.

Corollary 3.5. The generating function for the total number of occurrences of the subword pattern 132 in all the partitions of $[n]$ with exactly $k$ blocks is given by

$$
\frac{x^{k+2}}{(1-x) \cdots(1-k x)} \sum_{j=1}^{k-1} \frac{\left(\begin{array}{l}
j \\
2
\end{array}\right) \frac{3-2(j+1) x}{3}}{1-(j+1) x} \text {. }
$$

Proof. Using the notation of Theorem 3.4, we define $G_{k}^{\prime}(x, a)=\left.\frac{d}{d y} G_{k}(x, y \mid a)\right|_{y=1}$ and $G_{k}^{\prime}(x)=\left.\frac{d}{d y} G_{k}(x, y)\right|_{y=1}$ and get

$$
G_{k}^{\prime}(a)=x G_{k}^{\prime}+x^{2}(k-a) \sum_{j=1}^{a-1} G_{k}(x, 1, j)+x^{2} \sum_{j=1}^{a-1} G_{k-1}(x, 1, j),
$$

for all $a=1,2, \ldots, k-1$. Using the facts that $G_{k}(x, 1 \mid a)=x G_{k}(x, 1)$ and $G_{k}(x, 1)=$ $\frac{x^{k}}{(1-x) \cdots(1-k x)}$, we have

$G_{k}^{\prime}(a)=x G_{k}^{\prime}+x^{3}(k-a)(a-1) G_{k}(x, 1)+x^{3}(a-1) G_{k-1}(x, 1)=x G_{k}^{\prime}+\frac{x^{k+2}(a-1)(1-a x)}{(1-x) \cdots(1-k x)}$.

Summing over all $a=1,2, \ldots, k-1$, we get

$$
G_{k}^{\prime}=k x G_{k}^{\prime}+x G_{k-1}^{\prime}+\frac{x^{k+2}}{(1-x) \cdots(1-k x)}\left(\begin{array}{c}
k-1 \\
2
\end{array}\right) \frac{3-2 k x}{3},
$$


which is equivalent to

$$
G_{k}^{\prime}=\frac{x}{1-k x} G_{k-1}^{\prime}+\frac{x^{k+2}}{(1-x) \cdots(1-k x)^{2}}\left(\begin{array}{c}
k-1 \\
2
\end{array}\right) \frac{3-2 k x}{3} .
$$

Iterating the above recurrence relation using the initial condition $G_{1}^{\prime}(x)=0$, we obtain the requested result.

Collecting the coefficients of the generating function in Corollary 3.5 yields the following explicit formula for the total number of occurrences of 132 .

Corollary 3.6. The total number of occurrences of the subword pattern 132 in all of the partitions of $[n]$ with exactly $k$ blocks is given by

$$
\left(\begin{array}{l}
k \\
3
\end{array}\right) S_{n-2, k}+\sum_{j=3}^{k}\left(\begin{array}{l}
j \\
3
\end{array}\right) f_{n, j}
$$

where $f_{n, j}=\sum_{i=3}^{n-k} j^{i-3} S_{n-i, k}$.

Similar arguments also apply to the pattern 231 .

Theorem 3.7. Let $G_{k}(a)=F_{231}(x, y \mid a)$ be the generating function for the number of partitions $\pi=\pi_{1} \cdots \pi_{n-1}$ a with exactly $k$ blocks according to the number occurrences of the subword pattern 231, and let $G_{k}=\sum_{a=1}^{k} G_{k}(a)=F_{231}(x, y, k)$. Then the generating function $G_{k}(a)$ satisfies the recurrence relation

$$
G_{k}(a)=x G_{k}+x^{2}(y-1) \sum_{j=a+1}^{k}(k-j) G_{k}(j)+x^{2}(y-1) \sum_{j=a+1}^{k-1} G_{k-1}(j)
$$

with the initial conditions $G_{k}(k)=x G_{k}+x G_{k-1}, G_{k}(k-1)=x G_{k}, G_{1}=G_{1}(1)=\frac{x}{1-x}$, $G_{2}(1)=\frac{x^{3}}{(1-x)(1-2 x)}, G_{2}(2)=\frac{x^{2}}{1-2 x}$ and $G_{2}=\frac{x^{2}}{(1-x)(1-2 x)}$.

Corollary 3.8. The generating function for the total number of occurrences of the subword pattern 231 in all the partitions of $[n]$ with exactly $k$ blocks is given by

$$
\frac{x^{k+1}}{(1-x) \cdots(1-k x)} \sum_{j=3}^{k} \frac{(j-2)\left(4 j(j-1) x^{2}+3(1-3 j) x+6\right)}{6(1-j x)} .
$$

Proof. Using the notation of Theorem 3.7, we define $G_{k}^{\prime}(x, a)=\left.\frac{d}{d y} G_{k}(x, y \mid a)\right|_{y=1}$ and $G_{k}^{\prime}(x)=\left.\frac{d}{d y} G_{k}(x, y)\right|_{y=1}$ and get

$$
G_{k}^{\prime}(a)=x G_{k}^{\prime}+x^{2} \sum_{j=a+1}^{k}(k-j) G_{k}(x, 1, j)+x^{2} \sum_{j=a+1}^{k-1} G_{k-1}(x, 1, j),
$$


for all $a=1,2, \ldots, k-1$. Using the facts that $G_{k}(x, 1 \mid a)=x G_{k}(x, 1)$ and $G_{k}(x, 1)=$ $\frac{x^{k}}{(1-x) \cdots(1-k x)}$, we have

$G_{k}^{\prime}(a)=x G_{k}^{\prime}+\frac{\left(\begin{array}{c}k-a \\ 2\end{array}\right) x^{k+3}}{(1-x) \cdots(1-k x)}+\frac{(k-1-a) x^{k+2}}{(1-x) \cdots(1-(k-1) x)}+\frac{x^{k+1}}{(1-x) \cdots(1-(k-2) x)}$.

Summing over all $a=1,2, \ldots, k-2$ using the initial conditions $G_{k}^{\prime}(k)=x G_{k}^{\prime}+x G_{k-1}^{\prime}$ and $G_{k}^{\prime}(k-1)=x G_{k}^{\prime}$, we have

$$
G_{k}^{\prime}=\frac{x}{1-k x} G_{k-1}^{\prime}+\frac{x^{k+1}}{(1-x) \cdots(1-k x)} \frac{(k-2)\left(4 k(k-1) x^{2}+3(1-3 k) x+6\right)}{6(1-k x)} .
$$

Iterating the above recurrence relation using the initial condition $G_{2}^{\prime}(x)=0$, we obtain the requested result.

Equivalently, we also have the following explicit formula.

Corollary 3.9. The total number of occurrences of the subword pattern 231 in all of the partitions of $[n]$ with exactly $k$ blocks is given by

$$
2\left(\begin{array}{l}
k \\
3
\end{array}\right) S_{n-2, k}+\left(\begin{array}{c}
k-1 \\
2
\end{array}\right) S_{n-2, k-1}+\sum_{j=3}^{k}\left(\begin{array}{l}
j \\
3
\end{array}\right) f_{n, j}
$$

where $f_{n, j}=\sum_{i=3}^{n-k} j^{i-3} S_{n-i, k}$.

Combinatorial proofs may be given for Corollaries 3.3, 3.6, and 3.9 similar to those given in the second section, the details of which we leave to the interested reader. We were unable to find an explicit formula for the generating function in the cases 121, 132 , and 231, which we leave as open questions. The table below summarizes our results.

\begin{tabular}{c|l||c|l||c|l}
$\tau$ & Theorem & $\tau$ & Theorem & $\tau$ & Theorem \\
\hline \hline 111 & 2.1 & 132 & 3.4 & 221 & 2.7 \\
112 & 2.3 & 211 & 2.7 & 231 & 3.7 \\
121 & 3.1 & 212 & 2.9 & 312 & 2.13 \\
122 & 2.3 & 213 & 2.11 & 321 & {$[8]$} \\
123 & {$[8]$} & & & &
\end{tabular}

Table 1: Three letter subword patterns. 


\section{References}

[1] A. Burstein and T. Mansour, Counting occurrences of some subword patterns, Discr. Math. Theoret. Comp. Sci. 6 (2003) 1-12.

[2] W. Chen, E. Deng, R. Du, R. Stanley, and C. Yan, Crossings and nestings of matchings and partitions, Trans. Amer. Math. Soc. 359 (2007) 1555-1575.

[3] S. Elizalde and M. Noy, Consecutive patterns in permutations, Adv. Appl. Math 30 (2003) 110-125.

[4] S. Heubach and T. Mansour, Enumeration of 3-letter patterns in compositions, Integers Conference, Celebration of the 70th Birthday of Ron Graham, University of West Georgia Carrollton, 2005.

[5] S. Heubach and T. Mansour, Combinatorics of Compositions and Words, CRC Press, Boca Raton, 2009.

[6] V. Jelínek and T. Mansour, On pattern-avoiding partitions, Elect. J. Combin. 15 (2008) \#R39.

[7] M. Klazar, On abab-free and abba-free set partitions, Europ. J. Combin. 17 (1996) 53-68.

[8] T. Mansour and A. Munagi, Enumeration of partitions by long rises, levels and descents, J. Integer Seq. 12 (2009) Article 09.1.8.

[9] T. Mansour and S. Severini, Enumeration of $(k, 2)$-noncrossing partitions, Discr. Math. 308 (2008) 4570-4577.

[10] T. Mansour and B. Sirhan, Counting $\ell$-letter subwords in compositions, Discr. Math. Theoret. Comp. Sci. 8 (2006) 285-298.

[11] S. Milne, A q-analog of restricted growth functions, Dobinski's equality, and Charlier polynomials, Trans. Amer. Math. Soc. 245 (1978) 89-118.

[12] B. Sagan, Pattern avoidance in set partitions, preprint.

[13] R. Stanley, Enumerative Combinatorics, Vol. 1, Cambridge University Press, Cambridge, UK, 1996.

[14] D. Stanton and D. White, Constructive Combinatorics, Springer, New York, 1986. 